zierung, hat sich bewährt. Wer von Leistungen des Bundes profitieren will, muß einen angemessenen Eigenbeitrag leisten. Der Bund vermittelt Anstöße, mobilisiert Kräfte in den Regionen und trägt zu ihrer nationalen Bündelung bei. Dabei orientiert er sich nicht an einem einheitlichen Bundessatz, sondern an Leistungskriterien, dieflexibel gehandhabt werden können. Bundesleistungen an Vorhaben im Rahmen der regionalpolitisch ausgerichteten Technologiepolitik sollten u. a. davon abhängig gemacht werden, ob diese großräumig koordiniert und abgestimmt sind und eine wirksame "Ausstrahlung» in die Region erzielen. Allerdings muß der Bund gerade in finanzpolitisch schwierigen Zeiten ein verläßlicher Partner sein und Kontinuität in finanzieller Hinsicht gewährleisten.

- Mix von Basisfinanzierung und Förderung von guten, anwendungsorientierten Projekten: Der Bund soll einen angemessenen Teil der Basisfinanzierung von Teilnehmern an einem Technologieverbund übernehmen. Ein substantieller Teil seiner Unterstützung soll jedoch über die Förderung von guten, anwendungsorientierten Projekten von Teilnehmern aus dem Verbund geschehen. Dabei müssen Kriterien der Förderinstanzen des Bundes konkret der Kommission für Technologie und Innovation (KTI, vormals KWF) - erfüllt werden.
Projektorientiertes Vorgehen und Projektmanagement sollen nicht nur für F \& E gelten, sondern auch im Ausund Weiterbildungsbereich: der Bund sollte vermehrt eine Leistungsorientierung für Institutionen durch stärkeren Einsatz von projektbezogener Finanzierung anstreben. Damit sollen Wettbewerb und ein unternehmerischer Geist in die Schulen und F \& E-Einrichtungen getragen werden.

Das Netzwerk von Unternehmen, Hochschulen, Fachhochschulen, Technologie-Kompetenzzentren sowie Innovationsberatungsstellen und Technologieparks ist vertikal und horizontal weiter zu knüpfen. Klare regionale Knoten sollen vom Bund durch projektweise Förderung von $\mathrm{F} \& \mathrm{E}$ gestärkt werden. Damit soll das vorhandene und schlummernde Potential in den Regionen mobilisiert und ein Klima der offensiven Kooperation geschaffen werden. Gelingt es, ein solches Netzwerk aufzubauen, mit Leben zu füllen und das regulatorische Umfeld wesentlich zu verbessern, so ist die Chance deutlich größer, daß sich der Industriestandort Schweiz im internationalen Wettbewerb behaupten kann. Die Technologiepolitik leistet damit einen substantiellen Beitrag zur Erhaltung und Schaffung von attraktiven Arbeitsplätzen und Einkommen am Standort Schweiz.

\title{
Das Berggebiet und die Neuorientierung der schweizerischen Regionalpolitik
}

Vier Eigenschaften charakterisieren die Neuorientierung der schweizerischen Regionalpolitik: Das Berggebiet bleibt primäres Zielgebiet der meisten regionalpolitischen Instrumente, die kleinräumige (IHG-Regionen) wird durch eine großräumige (Kantone, Grenzregionen) Regionalpolitik ergänzt, zu den kohäsionspolitischen Instrumenten (IHG, REGIO PLUS) kommen integrationspolitische (INTERREG) hinzu, und der nachhinkende Disparitätenabbau soll durch die Förderung der regionalen Anpassungs- und Wettbewerbsfähigkeit abgelöst werden.

Diese Zieloptik ist konsistent mit der marktwirtschaftlichen Erneuerung des Wirtschaftsstandortes Schweiz als Antwort auf die globalwirtschaftlichen Herausforderungen. Die rasche Vervielfachung qualifizierter und differenzierter Standorte für die Wirtschaft und die neuen wirtschaftspolitischen Vorgaben durch die GATT/WTORegeln sowie die fortschreitende Integration großer
Wirtschaftsräume (Triade: Europa, Nordamerika, Südostasien) erfordern innovationsfördernde Rahmenbedingungen und wettbewerbsfähige Standorte in der Schweiz.

Die Regionalpolitik soll in erster Linie die wirtschaftliche Erneuerungsfähigkeit unterstützen, indem sie für diese Aufgaben geeignete Trägerschaften und Projekte mitfinanziert sowie offene und vernetzte Regionsstrukturen fördert. In diesem Dispositiv nehmen die Kantone als Träger der klein- und großräumigen Regionalpolitik eine Schlüsselrolle ein. Diese Position soll insbesondere durch die Neuordnung des bundesstaatlichen Finanz-

Paul Messerli, Prof. Dr., Geographisches Institut, Universität Bern, Hallerstraße 12, 3012 Bern 
ausgleiches gefestigt werden, indem der Handlungsspielraum der Kantone vergrößert, die Kooperationsmöglichkeiten erweitert und der Föderalismus gestärkt wurden. Die regionale Dimension bemißt sich im neuen Konzept der Regionalpolitik nach dem Prinzip der variablen Geometrie; regionale Zusammenarbeit und Vernetzung soll die aufgabenspezifische Funktions- und Wettbewerbsfähigkeit garantieren, im kleinräumigen sozial- und umweltpolitischen wie im großräumigen verkehrs- und standortpolitischen Bereich.

Dieser Artikel will am Beispiel des Berggebietes die regionalpolitische Neuorientierung konzeptuell und instrumentell erläutern und einer kritischen Würdigung unterziehen.

\section{Die schweizerische Regionalpolitik war weitgehend als Berggebietspolitik konzipiert}

Der Ursprung der schweizerischen Regionalpolitik ist im föderalistischen Aufbau und in der Vielgestaltigkeit der schweizerischen Eidgenossenschaft zu suchen. Die differenzierte Anwendung verschiedener staatlicher Maßnahmen, Sonder- und Vorzugsbehandlung bestimmter Gebiete und/oder Gruppen unseres Landes kennzeichnen seit je die Bundespolitik. Deutlich sichtbar wird dies beispielsweise in der Agrarpolitik, in der Verkehrspolitik oder beim Finanzausgleich. Die regionale Differenzierung orientierte sich von Anfang an am Berggebiet. Der Abwanderung aus der Berglandwirtschaft wurde bereits in den zwanziger Jahren mit der Agrarpolitik begegnet, dem Substanzverlust an Arbeitskräften, Einkommen und Steuermitteln und der Einschränkung des Handlungsspielraumes der Bergkantone und -gemeinden durch den 1959 eingeführten bundesstaatlichen Finanzausgleich. Die Grundidee, die sach- und sektoralpolitischen Maßnahmen des Bundes in die regionalpolitische Pflicht zu nehmen, ist so alt wie die öffentliche Wahrnehmung einer wirtschaftlichen Benachteiligung und Rückständigkeit des Berggebietes. Diese Doppelstrategie trägt aber den Zielkonflikt zwischen regionaler Ausgleichswirkung und sektoraler Allokationseffizienz stets in sich, was in Phasen der knappen öffentlichen Mittel immer wieder Anlaß zu Grundsatzdiskussionen über das regionalpolitische Instrumentarium gab.

Der Übergang von der impliziten zur expliziten Regionalpolitik kam 1974 auf den politischen Druck zustande, regionalpolitisch auf Bundesebene nicht nur sektoral, sondern gesamtwirtschaftlich zugunsten der strukturell, wachstumsdynamisch und wohlstandsmäßig benachteiligten Berggebiete (Peripherien) aktiv zu werden, aber auch um eine "Entflechtung» zwischen Allokations- und Ausgleichspolitik zu erwirken.

Der institutionelle Rahmen der neuen Berggebietspolitik wurde durch die Bildung der IHG-Regionen (heute 54) geschaffen. Ohne daß sie eine zusätzliche bundesstaatliche Hoheitsebene zwischen Gemeinden und Kantonen bilden, wurden sie zum institutionellen Träger der schweizerischen Regionalpolitik. Das Instrumentarium umfasste vier Elemente (HUBER, MORDASINI 1995):

(1) Das Bundesgesetz über Investitionshilfe für Berggebiete (IHG, 1974) zielte auf die Verbesserung der unternehmens- und haushaltsbezogenen Infrastruktur im Berggebiet und damit auf eine Erhöhung der Standortqualität. Mit dem Instrument der «Restfinanzierung» (maximal 25\% der Investitionskosten) sollten regionale Initiativen ausgelöst und koordiniert werden.

(2) Das Bundesgesetz über die Förderung des Hotel- und Kurortskredites (HKG, 1966/75) sollte die Erneuerung der traditionellen Hotelleriebetriebe, die zu zwei Dritteln aus der Zeit vor dem Zweiten Weltkrieg stammten, ermöglichen.

(3) Das Bundesgesetz über die Gewährung von Bürgschaften und Zinskostenbeiträgen in Berggebieten (BGB, 1976) diente der Kreditbeschaffung für Kleinund Mittelbetriebe mit beschränktem Zugang zu Risikokapital.

(4) Der Bundesbeschluß über Finanzierungsbeihilfen zugunsten wirtschaftlich bedrohter Regionen ( Bonny-Beschluß», 1978) war die Reaktion auf die Strukturkrise, die mit der Rezession (1974/75) in den traditionellen Industrieregionen des Berggebietes (Uhren, Textil, Metallindustrie) ausgelöst wurde.

Parallel dazu wurden in den Kantonen Programme zur regionalen Wirtschaftsförderung in Gang gesetzt, im Kanton Bern etwa auf der Grundlage des 1971 eingeführten Wirtschaftsförderungsgesetzes. Die Bindung der Finanzhilfen an die Erarbeitung eines regionalen Entwicklungskonzeptes löste im Berggebiet eine intensive Planungsphase aus, die vielerorts zum Ausgangspunkt einer neuen regionalen Bewußtseinsbildung wurde.

Mit diesem Schritt anerkannte der Bund die Notwendigkeit, dem Berggebiet einen «Sonderstatus» einzuräumen, sollte der Verfassungsauftrag der regionalen und sozialen Chancengleichheit und -gerechtigkeit eingelöst werden.

Im Rückblick auf zwanzig Jahre IHG-Praxis - das eigentliche Rückgrat der schweizerischen Regional- und Berggebietspolitik - sind drei Feststellungen zu machen:

1. Die Bildung von Regionen und die Ausarbeitung umfassender Entwicklungskonzepte haben nicht nur zu einer Verstärkung der überkommunalen Zusammenarbeit der Gemeinden des Berggebietes geführt, sondern auch zu einer Festigung des regionalen Denkens und Handelns beigetragen.

2. Unbestritten ist, daß die Investionshilfe (in über 5700 geförderten Projekten wurden mit rund 2 Mrd. IHGDarlehen und Zinskostenbeiträgen von über $13 \mathrm{Mrd}$. Schweizerfranken Investitionen ausgelöst) wesentlich dazu beigetragen hat, daß das Berggebiet insbesondere im Bereich der öffentlichen Grundversorgung heute über eine infrastrukturelle Ausstattung verfügt, die mit jener des Mittellandes weitgehend vergleichbar ist.

3. Weniger zufriedenstellend sind demgegenüber die Ergebnisse ausgefallen, was die Beschäftigungs- und 
Wohlstandsentwicklung sowie die Einkommenseffekte anbelangt. In diesen Bereichen konnte bei einer Vielzahl von Entwicklungsregionen der Rückstand gegenüber den wirtschaftlich starken Gebieten nicht aufgeholt werden.

Diese Aussage erstaunt nicht, wurde doch bereits Mitte der achtziger Jahre aus den beiden Nationalen Forschungsprogrammen «Regionalprobleme in der Schweiz" und "Sozioökonomische Entwicklung und ökologische Belastbarkeit im Berggebiet» festgehalten, daß in einer sich rasch ändernden wirtschaftlichen, technischen und gesellschaftlichen Umwelt jede Regionalpolitik zu spät komme, die einseitig auf den Abbau der eigenen Schwächen fixiert sei. Vielmehr gelte es, die regionalen Stärken rechtzeitig zu erkennen und in komparative Vorteile zu verwandeln (innovationsorientierte Regionalpolitik). Regionalpolitik dürfe ihre Aufgabe in den neunziger Jahren nicht mehr ausschließlich in der Vergangenheitsbewältigung sehen, sondern müsse sich viel stärker der Gestaltung der Zukunft durch gezielte Investitionen in die Fähigkeiten und Kompetenzen regionaler Entscheidungsträger und regionaler Unternehmer zuwenden. Weniger Ausgleichspolitik sei also für die neunziger Jahre gefragt, dafür mehr Prozeßpolitik.
2. Mit welchen Zukunftsproblemen sind die Berggebiete konfrontiert?

Während in den siebziger Jahren das schweizerische Berggebiet einen Bevölkerungsverlust von - 0,8\% erlitt, legte es in den achtziger Jahren mit $8,4 \%$ mehr als die Schweiz insgesamt (8\%) zu. Auch der Zuwachs von 15,8\% der Erwerbstätigen (Schweiz: 17,1\%) bestätigt die positive Gesamtbilanz für dieses Jahrzehnt. Eine detaillierte Analyse auf der Basis kommunaler und regionaler Entwicklungstypen (BÄTZING et al. 1995) deckt auf, daß dieses Wachstum sehr ungleich erfolgte, der sektorale Strukturwandel sich dem schweizerischen weitgehend annäherte, der Verflechtungsgrad der Gemeinden deutlich zunahm (die Zahl der isolierten Gemeinden ging auf $9 \%$ der Bergebietsgemeinden zurück) und die Siedlungsentwicklung durch Agglomerationsprozesse mit Funktionsentmischung von Wohnen und Arbeiten geprägt ist. So fallen $77 \%$ des Wachstums der Erwerbstätigen auf Pendlergemeinden und Zentren und nur 7\% auf Tourismusgemeinden. Entsiedlungsprozesse finden vor allem in den industriell geprägten Gemeinden und den Kleinstgemeinden mit weniger als 300 Einwohnern statt. Auf regionaler Ebene (IHG-Regionen, vgl. Karte) zeigt sich, daß die Zahl der zentrendominierten Regionen zwi-

\section{Regionale Entwicklungstypen der IHG-Regionen, 1990}

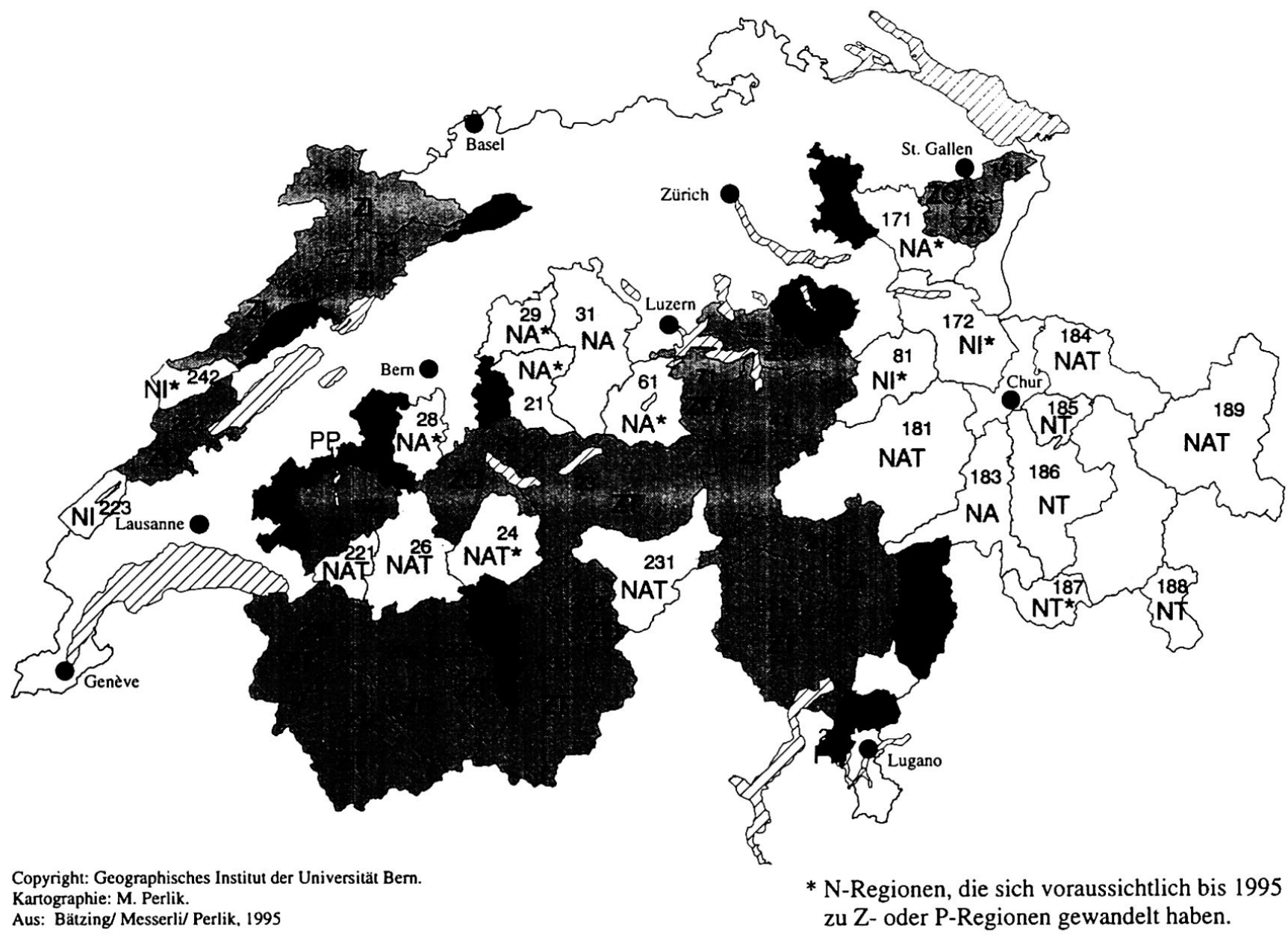


schen 1980 und 1990 deutlich zugenommen hat, die Pendlerregionen am Alpen- und Jurarand von den nahen Arbeitsplatzzentren und ihrer hohen Wohnstandortqualität profitieren und die nicht zentrendominierten (ländlichen) Regionen immer deutlicher das eigentliche "strukturschwache Berggebiet» ausweisen; insbesondere sind die Voraussetzungen für zentrenorientierte Tertiärisierungsprozesse ungünstig.

Was aus diesem Entwicklungsmuster insgesamt für die Zukunftsgestaltung entscheidend sein dürfte, ist die Tatsache, daß sich die Zentrenstruktur im Berggebiet verstärkt hat, wodurch die Allokationseffizienz künftiger Investitionen erhöht wird; dazu ist der Pendlerverflechtungsgrad so angestiegen, daß die interregionale Zusammenarbeit rasch an Bedeutung gewinnen wird. Die künftige Regionalpolitik muß dieser Entwicklung Rechnung tragen. Die Stärkung der Zentrenstruktur und die flexible Unterstützung der interregionalen Zusammenarbeit aufgrund von Nachbarschaften und bestehender Verflechtungen ist ein zwingender Ansatzpunkt.

Die achtziger Jahre brachten es an den Tag: Nur die Pendlerregionen am Alpen- und Jurarand können in größerem Maße vom Einkommensimport aus den Arbeitsplatzzentren außerhalb des Berggebietes profitieren. Die übrigen Regionen sind auf die eigenen Arbeitsplätze und Einkommensquellen angewiesen, um eine aktive Bevölkerung zu erhalten. Die beschleunigten strukturellen Anpassungsprozesse müssen in allen relevanten Beschäftigungsbereichen aus eigener Kraft bewältigt werden. Insbesondere für den Agrarsektor, die industriellen Betriebe und die Tourismusbranche verändern sich das marktliche Umfeld und die Konkurrenzverhältnisse in dramatischer Weise. Mit der Agrarpolitik 2002 wird auch die Berglandwirtschaft vermehrt in die unternehmerische Selbständigkeit entlassen. Der Tourismus verliert an Wettbewerbspositionen und sieht sich mit strukturellen und konjunkturellen Problemen konfrontiert. Mit dem Rückzug des Bundes aus dem Berggebiet (Armeereform, PTT-Reform, Verkehrspolitik) verliert das Berggebiet wichtige industrielle Arbeitsplätze. Die knappen öffentlichen Finanzen lassen trotz politischem Reformwillen für regionalpolitische und strukturpolitische Maßnahmen kaum einen größeren Mitteleinsatz erwarten.

Kann die Regionalpolitik in dieser Situation neue Akzente setzen? Sie kann es sicher nur in sehr beschränktem Maße, weil die Mittelausstattung äußerst bescheiden bleibt und die regionalpolitischen Akzente nach wie vor durch die sektoralpolitischen Entscheide gesetzt werden (Finanzausgleich, Agrarpolitik, Energiepolitik/Wasserzinsen, Verkehrspolitik usw.). Die Neuorientierung kann aber die bisher kleinräumig ausgleichsorientierte auf eine großräumigere anpassungs- und entwicklungsorientierte Regionalpolitik öffnen und sich mit den beschränkten Mitteln auf impulssetzende Programme und Projekte konzentrieren.

\section{Neuorientierung der Regionalpolitik als Rahmen für die künftige Berggebietspolitik}

Für eine Neuorientierung der Regionalpolitik des Bundes standen drei Gründe im Vordergrund: Neben den internationalen Herausforderungen (Öffnung der Märkte und internationaler Handel, EU-Politik, technologischer Wandel, Wettbewerbsfähigkeit der Standorte in der Schweiz) sind es die notwendigen Reformen und politischen Initiativen im Innern (Armeereform, neue Agrarpolitik, Technologiepolitik, neue Aufgabenverteilung zwischen Bund und Kantonen usw.), die eine generelle Überprüfung der regionalpolitischen Instrumente notwendig machten. Dazu kommt, daß wichtige regionalpolitische Erlasse Mitte der neunziger Jahre ausliefen und somit eine Anschlußgesetzgebung notwendig wurde. Die Grundzüge der Neuorientierung der schweizerischen Regionalpolitik wurden eingangs skizziert. Die Reformarbeiten standen von Anfang an unter dem Verdikt der Etappierung und verschiedener Verantwortungs- und Zuständigkeitsbereiche. Selbst unter dem Dach des BIGA beschäftigen sich mehrere Abteilungen mit regionalpolitischen Instrumenten. Ein konsistentes Gebäude entsteht nicht leicht, wenn tragende Pfeiler (etwa die Neuordnung des bundesstaatlichen Finanzausgleiches) durch andere «Firmen» erstellt werden. Trotzdem sind die Konturen des Gebäudes heute erkennbar (KIND 1995; Neuorientierung der Regionalpolitik, 1996),

\begin{tabular}{|c|l|l|}
\hline Massstab & $\begin{array}{l}\text { Grossräumig } \\
\text { - Grenzregionen } \\
\text { Kantone und } \\
\text { Kantonsgruppen }\end{array}$ & $\begin{array}{l}\text { Kleinräumig } \\
\text { - Gemeindeverbindungen } \\
\text { IHG-Regionen und } \\
\text { Erweiterungen }\end{array}$ \\
\hline Ausgleich & $\begin{array}{l}\text { Neuordnung } \\
\text { bundesstaatlicher } \\
\text { Finanzausgleich }\end{array}$ & $\begin{array}{l}\text { Horizontaler Nutzen- } \\
\text { und Lastenausgleich }\end{array}$ \\
\hline Allokation & Wasserzinserhöhung & $\begin{array}{l}\text { Agrarpolitik 2002 } \\
\text { (Kostenbeiträge, } \\
\text { Direktzahlungen) }\end{array}$ \\
\hline Kooperation & INTERREG II & $\begin{array}{l}\text { BWE } \\
\text { IHG }\end{array}$ \\
\hline
\end{tabular}

$$
\begin{array}{ll}
\text { BGB } & =\begin{array}{l}
\text { Bundesgesetz über die Gewährung von Bürgschatten und Zinskosten- } \\
\text { beiträgen in Berggebieten }
\end{array} \\
\text { BWE } & =\text { Bundesbeschluss zugunsten wirtschaftlicher Erneuerungsgebiete } \\
\text { HKG } & =\text { Bundesgesetz über die Förderung des Hotel- und Kurortkredites } \\
\text { IHG } & =\text { Bundesgesetz über Investitionshille für Berggebiete } \\
\text { REGIO PLUS }= & \begin{array}{l}
\text { Bundesbeschluss über die Unterstützung des Strukturwandels im } \\
\text { ländlichen Raum }
\end{array} \\
\text { INTERREG II }=\begin{array}{l}
\text { Bundesbeschluss über die Förderung der grenzüberschreitenden Zu- } \\
\text { sammenarbeit von Kantonen und Regionen }
\end{array}
\end{array}
$$

Abb.1 Dispositiv der groß- und kleinräumigen Regionalpolitik. Zuordnung der wichtigsten Instrumente zu den Feldern der Ziel-Maßstab-Matrix.

(kursiv: in Vorbereitung, Vernehmlassung oder parlamentarischer Beratung, Stand März 1996) 
und einzelne Zimmer des erweiterten Gebäudes sind bereits möbliert.

In der Unterscheidung zwischen einer groß- und kleinräumigen Regionalpolitik wird die Absicht des Bundes sichtbar, seine regionalpolitische Tätigkeit auf die größere Maßstabsebene zu verlagern. Die kleinräumige soll in die Kompetenz der Kantone abgegeben werden, ohne daß sich der Bund aus der finanziellen Verantwortung zieht. Die wichtige Aufbauarbeit bei der Schaffung funktionsfähiger regionaler Strukturen zur Bewältigung der zahlreichen überkommunalen Aufgaben soll damit nicht gefährdet werden. Mit der klaren Trennung der Zielbereiche soll erreicht werden, daß der regionalpolitische Mitteleinsatz transparenter und effizienter erfolgen kann.

Der beabsichtigte Schulterschluß der Regionalpolitik und der Raumplanung in einer gemeinsamen Raumordnungspolitik des Bundes soll dieses Gebäude tragen. Die neu zu schaffende Raumordnungskonferenz soll als Koordinationsorgan die bisher unbefriedigende Abstimmung der Sektoralpolitiken mit den raumordnungspolitischen Zielen vornehmen. Kosten und Nutzen dieser Verschränkung sind dabei klar auszuweisen.

Nach dem bisherigen Stand der parlamentarischen Beratung ist dieses Gebäude im Grundsatz nicht gefährdet. Entscheidend wird die konkrete Ausgestaltung des neuen Finanzausgleiches sein, und mit der Wasserzinserhöhung wird dann ein zusätzlicher jährlicher Finanzfluß in die Bergkantone ausgelöst, der mit ca. 130 Mio. Franken deutlich höher ausfällt als die regionalpolitischen $\mathrm{Fi}$ nanzhilfen von durchschnittlich 100 Mio. Franken pro Jahr.

\section{Was bedeutet die Neuorientierung der Regionalpolitik für die Berggebiete?}

Vorerst einmal Kontinuität, denn mit der Revision des IHG wird die von den Mitteln her am besten ausgestattete regionalpolitische Maßnahme im angestammten Perimeter fortgesetzt. Mit der Überführung des Bonny-Beschlusses (1978) in den Bundesbeschluß für wirtschaftliche Erneuerungsgebiete (1996) kommen auch neue Teile des Berggebietes (Unterwallis, Waadt, Zentralschweiz, Tessin) in den Genuß von Bundesbeiträgen zur Förderung innovativer Industrie- und Dienstleistungsbetriebe.

Neu wird mit REGIO PLUS ein Instrument geschaffen, das zusätzlich zu den IHG-Vorhaben im Berggebiet innovative Projekte und Programme zur Bewältigung des Strukturwandels speziell in nicht zentrenbezogenen Regionen (NA, NAT, NI, vgl. Karte) fördern will. Die dazu benötigten Mittel werden aber voraussichtlich dem IHGFonds belastet. Neu ist auch die Möglichkeit, daß Bergund Grenzkantone über Interreg Bundesbeiträge für die grenzüberschreitende Zusammenarbeit mit EU-Staaten anfordern können. Diese Verordnung ist seit 1995 in Kraft und dürfte etwa für die Region Chablais/Montblanc und Jura/Franche-Comté von Bedeutung sein.

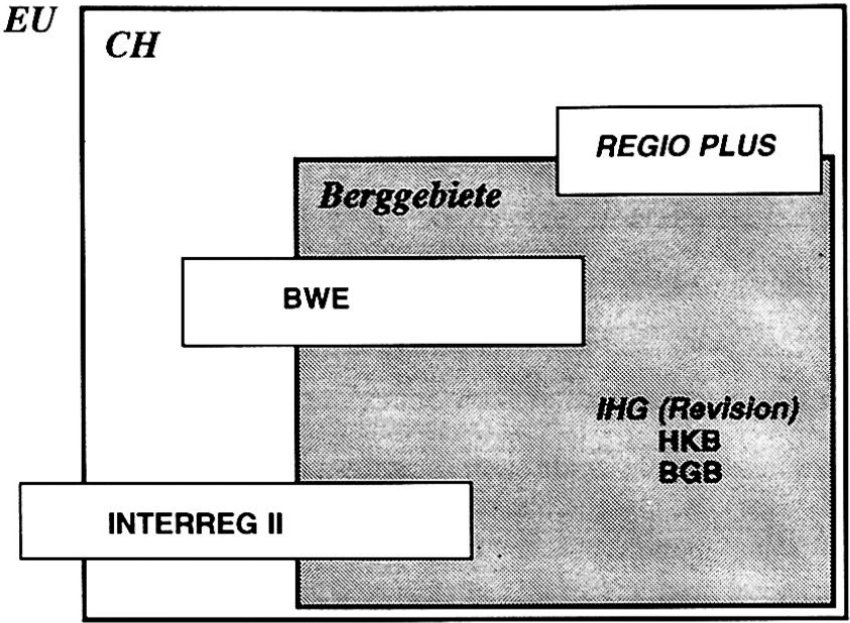

Abb.2 Territoriale Zuordnung der regionalpolitischen Instrumente des Bundes.

(kursiv: in parlamentarischer Beratung, Stand März 1996)

Mit der inhaltlichen Neugestaltung des IHG (Totalrevision) wird aber in der Berggebietsförderung wesentlich mehr Eigenverantwortung der Kantone, mehr Wettbewerb zwischen den Regionen und mehr Kreativität bei der Bewältigung der Strukturprobleme gefordert. Durch die Abkehr vom Restfinanzierungskriterium (max. 25\%) werden die Unterstützungsleistungen flexibler und können vor allem konzentrierter erfolgen. Mit der Abkehr vom Quotensystem zugunsten mehrjähriger Kreditlimiten an die Kantone soll der Wettbewerb verstärkt werden. Durch die sachliche und räumliche Schwerpunktbildung sowie die Gewährung von Pauschaldarlehen soll schließlich die Allokationseffizienz verbessert werden. Die zukunftsorientierte Stärkung der IHG-Regionen verschiebt sich so zugunsten der entwicklungsorientierten Infrastruktur, innovationsorientierter Projekte und der interregionalen Zusammenarbeit.

REGIO PLUS ist ganz nach dem Vorbild der OECD-Politik für den ländlichen Raum konzipiert. Es hat zwar einen geographischen Geltungsbereich (das ländliche Gebiet, auch außerhalb des IHG-Perimeters), fördert aber keine Infrastrukturen, sondern neuartige Vorhaben organisatorischer, konzeptioneller und institutioneller Natur. Im Vordergrund stehen Kooperationen zwischen Privaten und der öffentlichen Hand, etwa im Bereich des ländlichen Tourismus, oder branchenübergreifende Kooperationen der Industrie und des Gewerbes. Der Bund will Impulse auslösen, unterstützt professionelles Management und entläßt solche Projekte nach einer gewissen Zeit in die Selbständigkeit. Über ein nationales Netzwerk will er zudem den Informations-, Wissens- und Erfahrungsaustausch sicherstellen. Dieses Instrument ist für zehn Jahre mit 70 Mio. Franken ausgestattet.

So bescheiden die Reform aussehen mag, sie ist konzeptuell und instrumentell richtig angelegt. Die knappen 
Mittel werden zukunftsorientiert eingesetzt, die Anreizstruktur der Mittelvergabe stimuliert innovative Projekte, und die Konzentrationsmöglichkeit des Mitteleinsatzes gewährleistet bessere Wirkungen. Die gezielte projekt- und betriebsorientierte Kooperationsförderung des REGIO PLUS ist als experimenteller Teil der neuen Regionalpolitik zu verstehen und dürfte aufgrund ausländischer Erfahrungen innovative Kräfte mobilisieren und koordinieren.

Insgesamt fließen dem Berggebiet durch diese Instrumente nicht mehr Mittel zu. Erst die Neuausgestaltung des Finanzausgleiches und die definitive Entscheidung über die Erhöhung der Wasserzinsen werden jenen Mittelzufluß bestimmen, über den das Berggebiet dann zusätzlich selber verfügen kann.

Vorläufig aber beobachtet die schweizerische Arbeitsgemeinschaft für die Berggebiete (SAB) mit Sorge, wie sich der Bund und zum Teil die Kantone trotz Neuorientierung der Regionalpolitik aus dem Berggebiet zurückziehen. Die linearen Subventionskürzungen im Zuge der Budgetkorrekturen, die Kürzung der Schlechtwetterentschädigungen, der massive Abbau im öffentlichen Verkehr und die Streichung der Bundesbeiträge zugunsten des Viehabsatzes aus dem Berggebiet sind Beispiele dafür.

Wir befinden uns in einer Phase des Übergangs von der kleinräumigen zu der großräumigen Regionalpolitik des Bundes. Konzeptuell ist dieser Schritt angelegt, instrumentell aber nur ansatzweise vollzogen. Solange über die materiell entscheidenden Instrumente wie den neuen bundesstaatlichen Finanzausgleich oder die Wasserzinsregelung politisch nicht entschieden ist, bleibt dieser Schrittwechsel weitgehend Absicht. Die Doppelstrategie gibt auf der andern Seite die Möglichkeit, auf beiden Ebenen neue Erfahrungen zu sammeln und den regionalpolitischen Lernprozeß auszuweiten.

\section{Literatur}

BÄTZING, W., MESSERLI, P., PERLIK, M. (1995): Regionale Entwicklungstypen. Analyse und Gliederung des schweizerischen Berggebietes. Biga/Zentralstelle für regionale Wirtschaftsförderung. EDMZ, Bern.

BRUGGER, E. A., FURRER, G., MESSERLI, B., MESSERLI, P. (1984): Umbruch im Berggebiet. Les régions de montagne en mutation. P. Haupt, Bern.

BRUGGER, E. A., FREY, R. L. (1985): Regionalpolitik Schweiz: Ziele, Probleme, Erfahrungen, Reformen. P. Haupt, Bern.

HUBER, S., MORDASINI, R. (1994): Das Investitionshilfegesetz ist volljährig. Volkswirtschaft 7: 18-27. Eidg. Volkswirtschaftsdepartement, Bern.

KIND, V. (1995): Neuorientierung der Regionalpolitik. Volkswirtschaft 1: 13-19. Eidg. Volkswirtschaftsdepartement, Bern.

MESSERLI, P.(1991): Herausforderungen und Bedrohungen des schweizerischen Berggebietes durch Europa an der Wende zum 21. Jahrhundert. Bätzing/Messerli (Hrsg.): Die Alpen im Europa der 90er Jahre. Geographica Bernensia P 22: 142-176.

Neuorientierung der Regionalpolitik (1996): Botschaft an das Parlament. Eidg. Volkswirtschaftsdepartement, Bern.

REY, A. (1993): Finanzreformen für die Schweiz. Schriftenreihe der Fachgruppe für kantonale Finanzfragen, Band 3. KDMZ, Zürich.

ROREP (1992): Im Hinblick auf Europa 1993: Vorschläge für eine strategische schweizerische Regionalpolitik. Peter Lang.

STUCKI E. W., BORTHIOT UTZMANN, E. (1994): Agriculture et zones rurales: une comparaison des politiques structurelles de l'Union européenne et de la Suisse. IER, ETH, Zürich.

THIERSTEIN, A., EGGER, U.K. (1994): Integrierte Regionalpolitik. Ein prozeßorientiertes Konzept für die Schweiz. Schweizerisches Institut für Außenwirtschafts-, Struktur- und Regionalforschung an der HSG, Band 32. Verlag Rüegger.

WACHTER, D. (1995): Schweiz - eine moderne Geographie. Verlag NZZ, Zürich. 